\title{
Clinical Study \\ Screening for Bipolar Disorder Symptoms in Depressed Primary Care Attenders: Comparison between Mood Disorder Questionnaire and Hypomania Checklist (HCL-32)
}

\author{
Anna Sasdelli, Loredana Lia, C. Claudia Luciano, Claudia Nespeca, Domenico Berardi, and \\ Marco Menchetti \\ Institute of Psychiatry, University of Bologna, Viale C. Pepoli 5, 40123 Bologna, Italy
}

Correspondence should be addressed to Marco Menchetti; marco.menchetti3@unibo.it

Received 14 December 2012; Revised 10 March 2013; Accepted 14 March 2013

Academic Editor: Joanna Rymaszewska

Copyright (C) 2013 Anna Sasdelli et al. This is an open access article distributed under the Creative Commons Attribution License, which permits unrestricted use, distribution, and reproduction in any medium, provided the original work is properly cited.

\begin{abstract}
Objective. To describe the prevalence of patients who screen positive for bipolar disorder (BD) symptoms in primary care comparing two screening instruments: Mood Disorders Questionnaire (MDQ) and Hypomania Checklist (HCL-32). Participants. Adult patients presenting to their primary care practitioners for any cause and reporting current depression symptoms or a depressive episode in the last 6 months. Methods. Subjects completed MDQ and HCL-32, and clinical diagnosis was assessed by a psychiatrist following DSM-IV criteria. Depressive symptoms were evaluated in a subgroup with the Patient Health Questionnaire (PHQ-9). Results. A total of 94 patients were approached to participate and 93 completed the survey. Among these, $8.9 \%$ screened positive with MDQ and 43.0\% with HCL-32. MDQ positive had more likely features associated with BD: panic disorder and smoking habit $(P<.05)$. The best test accuracy was performed by cut-off 5 for MDQ (sensitivity $=.91$; specificity $=.67)$ and 15 for HCL-32 $($ sensitivity $=.64$; specificity $=.57)$. Higher total score of PHQ-9 was related to higher total scores at the screening tests $(P<.001)$. Conclusion. There is a significant prevalence of bipolar symptoms in primary care depressed patients. MDQ seems to have better accuracy and feasibility than HCL-32, features that fit well in the busy setting of primary care.
\end{abstract}

\section{Introduction}

Bipolar disorder (BD) has an estimated lifetime prevalence rate between $2 \%$ and $6 \%$ when wider range of bipolar spectrum disorders is considered [1]. It is a complex mood disorder frequently associated with medical and psychiatric comorbidity and high suicide rate [2]. Suicidal risk in BD is esteemed to be 15-20 times higher than the general population, and self-harm ideation is reported by $79 \%$ of patients $[3,4]$. Nevertheless, an average delay of 8-10 years from first onset of mood symptoms to a formal diagnosis of bipolar disorder occurs $[5,6]$. Longitudinal researches show that a patient is euthymic for half of the time, while manic or hypomanic symptoms are present only in the $12 \%$; in the rest of the time, a patient has depressive symptoms $[7,8]$. Hypomanic symptoms are often perceived as egosyntonic, while it is depression that usually leads the patient to the physician [9]. Thus, any loss or lack of information on hypomanic symptoms increases the bias in favour of a diagnosis of depression [10].

Primary care is the health service entry point for the majority of people suffering from depressive disorders and therefore could play a key role in the detection and management of BD. Although prevalence of symptoms and diagnosis of $\mathrm{BD}$ is elevated in depressed patients of primary care, Frye et al. found that $78 \%$ of primary care physicians (PCPs) failed to detect or misdiagnosed $\mathrm{BD}[11,12]$. To improve recognition of $\mathrm{BD}$, several rapid instruments have been developed in the last ten years, including the Mood Disorder Questionnaire (MDQ) and the Hypomania Checklist (HCL-32), now probably the most studied $[13,14]$. Both the instruments are validated in psychiatric outpatients settings, and guidelines suggest their usefulness in secondary care, while few studies assessed the employ in primary care [15]. 
Gorski et al. first used MDQ in primary care to test the association with principal complaints of patients referring to their PCP and found that participants who did complain of anxiety and depression had higher incidence of positive MDQ scores (16.4\%) [16, 17]. Das et al. found a lower prevalence $(9.8 \%)$ in low-income patients and confirmed the association with anxiety and depression, worse quality of life, and more functional impairment [18]. Consistently, a French study reported a similar value of prevalence $(8.3 \%)$ and a higher rate of positive screening among younger patients, separated, divorced, and unemployed [19]. Other studies conducted in specific populations, patients taking antidepressant, the presence of current indices of BD (depression, anxiety, and substance abuse) found higher prevalence rate ranging from $21.3 \%$ and $27.9 \%$ [20-22]. Only one study used HCL32 in a primary care setting in comparison with another screening instrument, the Bipolar Spectrum Disorders Scale, reporting the $28.27 \%$ of test positivity with the HCL-32 [23, 24].

In addition, no studies directly compared the two instruments MDQ and HCL-32 in the primary care setting, while some comparisons are available in studies on psychiatric outpatient services. All of them showed similar overall screening qualities of the two tests, and sensitivity of HCL32 was always slightly higher [25-30].

The objective of our study was to assess the prevalence of symptoms of the bipolar spectrum in primary care patients with current depression using the Mood Disorder Questionnaire (MDQ) and the Hypomania Checklist (HCL-32). In particular, we performed the first comparison between the two instruments in primary care.

\section{Method}

2.1. Setting and Participants. The present study was conducted in two primary care groups in Bologna in the first semester of 2011, located in the Borgo Panigale and Porto districts and included a total of 37 PCPs. In these groups, a psychiatric consultation-liaison project was implemented since 2001 and 2006, respectively. Furthermore, PCPs received a training about depressive disorder symptoms and DSM-IV diagnosis and criteria in 2009.

PCPs were asked to refer all patients aged 18 or more they visited in the study period and reported clinically relevant depressive symptoms or suffered from a depressive episode. Exclusion criteria were refusal to receive a psychiatric consultation or to participate in the study, inability to read or write, medical illness that would prevent completion of the interview, previous diagnosis of bipolar disorder, psychotic disorders, and mental retardation or cognitive impairment.

2.2. Diagnosis of Mood Disorder. The diagnosis of current or past 6-month mood disorder was subsequently performed by a consultant psychiatrist of the Bologna Psychiatric Consultation-liaison Service. The psychiatrist followed DSMIV criteria to formulate the diagnosis of mood disorder and was blind from results of MDQ and HCL-32.

Patients referred by the Primary Care Group Porto also received the Patient Health Questionnaire (PHQ-9), a validated tool, composed by 9 item, corresponding to DSMIV diagnostic criteria for major depressive episode [31]. The score is from 0 to 3 for each question; thus, the total score can range from 0 to 27. A higher score indicates greater depression: a patient score of 10 or greater suggests a diagnosis of MDD.

2.3. Bipolar Spectrum Symptoms Evaluation. Patients with a current or past (precedent 6 month) diagnosis of major depressive episode were asked to complete the MDQ and the HCL-32 to assess the prevalence of symptoms of the bipolar spectrum. Italian versions of the two instruments are available after studies of validation conducted in psychiatric outpatient services [14, 25, 32].

The MDQ is a self-report questionnaire composed by 17 questions: 13 yes/no items on the symptoms derived from the Diagnostic and Statistical Manual of Mental Disorder (DSM-IV) criteria and 4 questions about the cooccurrence of symptoms, levels of functioning, familiar history of $\mathrm{BD}$, and previous diagnosis of $\mathrm{BD}$. A positive MDQ screen is defined as endorsement of at least 7 or more symptoms items, cooccurrence of two or more symptoms, and moderate or severe impairment (MDQ standard cut-off) [13]. We also used another cut-off of 6 items without criteria of cooccurrence and functioning, as suggested by Hardoy et al. in the validation of the Italian MDQ for single-step studies in psychiatric outpatients [32].

The HCL-32 consists of 32 yes/no statements regarding a period when the patient remembers he was in a "high" mood. Items ask whether specific behaviours (e.g., "I spend more money/too much money"), thoughts (e.g., "I think faster"), or emotions (e.g., "my mood is significantly better") were present in such a state. Higher scores reflect more severe hypomanic states. The HCL-32 standard cut-off is represented by the endorsement of at least 14 items or more. We also analyzed the cut-off of 12, proposed by Carta for single-step researches in an outpatient population [25].

In addition, data about sociodemographics, medical history, family history of psychiatric disorders, and current and past psychotropic medication were collected using specific forms.

2.4. Statistical Analyses. Patients screened as positive with the considered instruments were compared with those screened as negative. Chi-square test $\left(\chi^{2}\right)$ was used to compare the frequency of categorical variables between groups: frequency of positive and negative screenings and sociodemographic and clinical features. T-test was used to compare the means of continuous variables between two or more groups. Correlations between ordered variables as PHQ-9, HCL-32, and MDQ total score were assessed with Pearson linear correlation $(\rho)$. The accuracy of the two screening instruments was calculated in terms of sensitivity and specificity for each possible cut-off point of the scales, considering as cut-off only the number of positive answers, in particular for MDQ no adjunctive criteria were comprised. Performance of the scales was assessed by means of the Receiver Operating Characteristic (ROC) Analysis [33]. Data were analyzed by using SPSS for Windows, version 17.0. 
TABLE 1: MDQ-positive patients (standard cut-off ${ }^{*}$ ) and diagnosis of BD.

\begin{tabular}{lcc}
\hline & BD- & BD+ \\
\hline MDQ- & 52 & 9 \\
MDQ+ & 4 & 2
\end{tabular}

*Standard cut-off: endorsement of at least 7 or more symptoms items, cooccurrence of two or more symptoms, and moderate or severe impairment. MDQ: mood disorder questionnaire; BD: bipolar disorder.

TABLE 2: HCL-32 positive patients (standard cut-off*) and diagnosis of BD.

\begin{tabular}{lcc}
\hline & BD- & BD+ \\
\hline HCL-32- & 36 & 5 \\
HCL-32+ & 20 & 6 \\
\hline
\end{tabular}

*Standard cut-off: endorsement of at least 14 or more items.

HCL-32: hypomania checklist; BD: bipolar disorder.

\section{Results}

3.1. Sociodemographic and Clinic Characteristics. Out of the 94 primary care attenders enrolled in the study, 93 completed the two questionnaires, and 67 received the diagnostic assessment of the consultant psychiatrist. The mean age of the participants was $49.1( \pm 15.1)$ years. The majority was female $(72.3 \%)$ and had a secondary education level or higher $(64.0 \%)$; forty-five point two percent were in a nonprofessional condition and in particular retired (18.0\%), housewives (10.6\%), unemployed (4.44\%), and students (4.2\%). Twenty point two percent of the sample was separated or divorced.

Eleven patients met criteria for BD II (11.7\%). As many as 29 participants had a concomitant anxiety disorder (30.9\%): 17 met criteria for generalized anxiety disorder (18.1\%) and 12 for panic disorder (12,8\%). Smoking habit was present in $30.0 \%$ of the sample, hypertension in $26.6 \%$. Twelve patients $(12.8 \%)$ reported the concomitance of two cardiovascular risk factors (we consider smoking habit, hypertension, dyslipidemia, obesity, and diabetes). About two-thirds of the patients were treated by PCPs with antidepressant drugs (67.0\%); out of these, $74.2 \%$ were represented by Selective Serotonin Reuptake Inhibitors, $16.1 \%$ by Serotonin and Noradrenalin Reuptake Inhibitors, and $9.7 \%$ by other antidepressants including tricyclics.

3.2. Diagnosis of BD and Screening Tests Scores. Patients meeting criteria for BD had a mean MDQ score of 6.18 $( \pm 1.85)$ and HCL-36 score of $15.36( \pm 4.90)$. Patients without diagnosis of BD had $3.10( \pm 2.77 ; P<.001)$ and $11.46( \pm 6.64$; $P=.069)$, respectively. Seven patients met criteria with a clinical diagnosis of $\mathrm{BD}$ and screened positive to both the two screening instruments, using standard cut-off. The MDQ positive and HCL-32 positive patients with confirmed or excluded BD diagnosis are summarized in Tables 1 and 2.

3.3. Symptoms Prevalence. Tables 3 and 4 summarize the endorsement rate of MDQ and HCL-32 items. In the sample affirmative responses to MDQ items ranged from $9.7 \%$ ("spending money got you or your family into trouble") to $50.5 \%$ ("had much more energy than usual"); HCL32 affirmative items responses ranged from 7.5\% ("drink more alcohol" and "take more drugs") to $76.3 \%$ ("feel more energetic and more active"). The symptoms elicited by 8 of the 13 items of the MDQ and 25 of the 32 items of the HCL-32 were more prevalent among participants screening positive than among those screening negative $(P<.05)$. The proportion of participants who met the MDQ diagnostic criteria for bipolar spectrum was $8.6 \%$ for the standard cutoff and $23.7 \%$ considering the less restrictive cut-off of 6 . The percentage of positive screening for history of hypomanic symptoms at the HCL-32 was $43.0 \%$ with 14 as cut-off and $55.9 \%$ for 12 or more items endorsed.

3.4. Characteristics Associated with MDQ+ and HCL-32+. The population identified by MDQ was part of the population that screened positive at the HCL-32. Characteristics associated to positivity to the two tests are presented in Tables 5 and 6 . Using the cut-off standard of MDQ, we find clinical features related to positive screening: panic disorder $(P=.029)$ and smoking habit $(P=.028)$. MDQ-positive patients are more likely to be smokers and accordingly had a higher cardiovascular risk. The less restrictive cut-off (6) did not find correlations, except for current AD therapy. The HCL-32 positive patients were younger, more likely to have a high level of instruction, and they are more likely smokers $(P<.05)$. There was no difference in gender between groups. With the standard cut-off (14), there was also a trend toward a higher incidence of positive screening in patients who were separated or divorced, but this did not reach statistical significance $(P=.06)$.

3.5. Sensitivity and Specificity. Performances of MDQ and HCL-32 are illustrated by ROC analysis (Figure 1), with the report of sensitivity and specificity for each cut-off. The best accuracy of the test is given by cut-off 5 for MDQ (sensitivity $=.91$; specificity $=.67$ ) and 15 for HCL-32 (sensitivity = .64; specificity $=.57)$.

3.6. Relationship with PHQ-9. Analysis conducted in a subsample ( $n=40$ ) who received the PHQ-9 showed that higher PHQ-9 score correlated with both higher MDQ and HCL-32 scores $(\rho=.316-P=.036 ; \rho=.530-P<.001$, resp.).

\section{Discussion}

In this study, we assessed the prevalence of symptoms ascribable to the spectrum of bipolar disorders through the use of two instruments meant for the screening of bipolar disorder (MDQ and HCL-32) in a clinical sample of primary care depressed patients. The two autosomministrated tests highlight the pattern of symptoms that can suggest an undiagnosed $\mathrm{BD}$, but without diagnostic properties. Eleven patients $(11,7 \%)$ met diagnostic criteria for $\mathrm{BD}$, all of them are described as type II bipolar disorder, while the prevalence 
TABLE 3: Frequencies of endorsed items to MDQ (standard cut-off ${ }^{*}$ ) and correlation with positive screening.

\begin{tabular}{|c|c|c|c|c|c|c|}
\hline & \multirow{2}{*}{$\begin{array}{c}\text { Item } \\
\text { endorsed, } \\
\% \\
\end{array}$} & \multicolumn{2}{|c|}{ MDQ } & \multirow[b]{2}{*}{ Chi-sq } & \multirow[b]{2}{*}{ df } & \multirow[b]{2}{*}{$P$} \\
\hline & & Positive, \% & Negative, \% & & & \\
\hline $\begin{array}{l}\text { (1) You felt so good/hyper that other people thought you were not your } \\
\text { normal self or you were so hyper that you got in trouble? }\end{array}$ & 16.1 & 37.5 & 14.1 & 2.955 & 1 & ns \\
\hline $\begin{array}{l}\text { (2) You were so irritable that you shouted at people or started fights or } \\
\text { arguments? }\end{array}$ & 30.1 & 62.5 & 27.1 & 4.365 & 1 & .037 \\
\hline (3) You felt much more self-confident than usual? & 43.0 & 100.0 & 37.6 & 11.598 & 1 & $<.001$ \\
\hline $\begin{array}{l}\text { (4) You got much less sleep than usual and found you did not really miss } \\
\text { it? }\end{array}$ & 29.0 & 87.5 & 23.5 & 14.523 & 1 & $<.001$ \\
\hline (5) You were much more talkative or spoke much faster than usual? & 23.7 & 75.0 & 18.8 & 12.777 & 1 & $<.001$ \\
\hline $\begin{array}{l}\text { (6) Thoughts raced through your head or you could not slow your mind } \\
\text { down? }\end{array}$ & 29.0 & 75.0 & 24.7 & 8.977 & 1 & .003 \\
\hline $\begin{array}{l}\text { (7) You were so easily distracted by things around you that you had } \\
\text { trouble concentrating or staying on track? }\end{array}$ & 38.7 & 75.0 & 35.3 & 4.859 & 1 & .028 \\
\hline (8) You had much more energy than usual? & 50.5 & 75.0 & 48.2 & 2.095 & 1 & ns \\
\hline (9) You were much more active or did many more things than usual? & 48.4 & 87.5 & 44.7 & 5.362 & 1 & .021 \\
\hline $\begin{array}{l}\text { (10) You were much more social or outgoing than usual; for example, } \\
\text { you telephoned friends in the middle of the night? }\end{array}$ & 12.9 & 12.5 & 12.9 & 0.001 & 1 & ns \\
\hline (11) You were much more interested in sex than usual? & 22.6 & 75.0 & 17.6 & 13.758 & 1 & $<.001$ \\
\hline $\begin{array}{l}\text { (12) You did things that were unusual for you or that other people might } \\
\text { have thought were excessive, foolish, or risky? }\end{array}$ & 11.8 & 25.0 & 10.6 & 1.456 & 1 & ns \\
\hline (13) Spending money got you or your family into trouble? & 9.7 & 12.5 & 9.4 & 0.080 & 1 & ns \\
\hline
\end{tabular}

*Standard cut-off: endorsement of at least 7 or more symptoms items, cooccurrence of two or more symptoms and moderate or severe impairment. MDQ: mood disorder questionnaire; Chi-sq: chi-square test; df: degrees of freedom; ns: nonsignificant.

of bipolar spectrum disorder symptoms was very different between the two instruments. When standard criteria to establish the test positivity were considered, MDQ was positive in the $8.6 \%$ of the sample, whereas HCL-32 in the $43.0 \%$.

Few studies were conducted in the primary care setting, and our MDQ positive rate appears to be consistent with values reported in previous researches recruiting not specific populations $[18,19]$. On the contrary, primary care studies on selected patients (mood or other psychiatric disorder and/or in treatment with antidepressant drugs) reported higher values, between 21.0 and $27.9 \%$ [20-22], similar to those found in studies on psychiatric outpatients services $[34,35]$. The lower prevalence of MDQ-positive reported in our sample may be due to the inclusion of patients with mild or subthreshold depressive symptoms, frequently observed in primary care.

A higher rate of positive screening was found with the HCL-32. We can compare this result with only one primary care study using the HCL-32 in a population of patients with major depressive disorder. However, this research used a more restrictive cut-off (18) and reported $28.3 \%$ of test positivity [24]. Our finding seems to be more comparable to those obtained in secondary care and in very selected samples $[27,36,37]$.

Considering the patients identified by the instruments, we found that MDQ positive patients were all included in the HCL-32 positive cases. HCL-32 test positivity identified some specific sociodemographic and clinical features: a younger age, a higher education level, and smoking habit. Consistently with previous studies $[18,34,38]$, MDQ test positivity was associated only with clinical characteristics including panic disorder and smoking habit; in literature, these characteristics are both strongly related to $\mathrm{BD}[39,40]$. In addition, we found an association with MDQ-positive patients with at least two cardiovascular risk factors. These described correlations did not reach statistical significance using a less restrictive cutoff of the MDQ (6). Our data collected in primary care seem to indicate that MDQ test positivity detects a more specific population of depressed patients inside a wider group identified by HCL-32.

The comparison in terms of psychometric proprieties seems to indicate a better accuracy of MDQ. We can also compare our findings with those obtained in one another Italian study that evaluated the two tests in a psychiatric setting [25]. Our results show lower values of sensitivity and specificity for both scales; however, MDQ sensitivity and specificity of the best performing cut-off remain similar to the results presented by Carta, while accuracy of HCL-32 is lower to a great extent. In particular, a less restrictive cut-off (5) of the MDQ showed better accuracy than the cut-off standard. The same trend of differences between MDQ and HCL-32 remains if we consider the other studies on the accuracy of the two instruments available in primary care $[20,24]$.

Considering these data, the rate of MDQ positive seems to be more consistent with the literature and with data 
TABLE 4: Frequencies of endorsed items to HCL-32 (standard cut-off ${ }^{*}$ ) and correlation with positive screening.

\begin{tabular}{|c|c|c|c|c|c|c|}
\hline & Item & & & & & \\
\hline & $\begin{array}{c}\text { endorsed, } \\
\% \\
\end{array}$ & Positive, \% & Negative, \% & Chi-sq & $\mathrm{df}$ & $P$ \\
\hline (1) I need less sleep & 45.2 & 28.3 & 67.5 & 14.143 & 1 & $<.001$ \\
\hline (2) I feel more energetic and more active & 76.3 & 64.2 & 92.5 & 10.144 & 1 & .001 \\
\hline (3) I am more self-confident & 67.7 & 50.9 & 90.0 & 15.913 & 1 & $<.001$ \\
\hline (4) I enjoy my work more & 41.9 & 34.0 & 52.5 & 3.217 & 1 & ns \\
\hline (5) I am more sociable (make more phone calls, go out more) & 58.1 & 41.5 & 80.0 & 13.870 & 1 & $<.001$ \\
\hline (6) I want to travel and/or do travel more & 37.6 & 24.5 & 55.0 & 9.018 & 1 & .003 \\
\hline (7) I tend to drive faster or take more risks when driving & 12.9 & 5.70 & 22.5 & 5.752 & 1 & .016 \\
\hline (8) I spend more money/too much money & 19.4 & 9.40 & 32.5 & 7.770 & 1 & .005 \\
\hline (9) I take more risks in my daily life & 19.4 & 15.1 & 25.0 & 1.433 & 1 & ns \\
\hline (10) I am physically more active (sport, etc.) & 59.1 & 41.5 & 82.5 & 15.850 & 1 & $<.001$ \\
\hline (11) I plan more activities or projects & 59.1 & 35.8 & 90.0 & 27.662 & 1 & $<.001$ \\
\hline (12) I have more ideas, I am more creative & 55.9 & 30.2 & 90.0 & 33.083 & 1 & $<.001$ \\
\hline (13) I am less shy or inhibited & 35.5 & 15.1 & 62.5 & 22.378 & 1 & $<.001$ \\
\hline (14) I wear more colourful and more extravagant clothes/make-up & 18.3 & 3.8 & 37.5 & 17.358 & 1 & $<.001$ \\
\hline (15) I want to meet or actually do meet more people & 62.4 & 37.7 & 95.0 & 31.848 & 1 & $<.001$ \\
\hline (16) I am more interested in sex, and/or have increased sexual desire & 38.7 & 17.0 & 67.5 & 24.522 & 1 & $<.001$ \\
\hline (17) I am more flirtatious and/or am more sexually active & 28.0 & 9.4 & 52.5 & 20.991 & 1 & $<.001$ \\
\hline (18) I talk more & 38.7 & 18.9 & 72.5 & 20.448 & 1 & $<.001$ \\
\hline (19) I think faster & 41.9 & 18.9 & 27.5 & 26.928 & 1 & $<.001$ \\
\hline (20) I make more jokes or puns when I am talking & 36.6 & 22.6 & 55.0 & 10.291 & 1 & .001 \\
\hline (21) I am more easily distracted & 22.6 & 18.9 & 27.5 & 0.972 & 1 & ns \\
\hline (22) I engage in lots of new things & 28.0 & 13.2 & 27.5 & 13.310 & 1 & $<.001$ \\
\hline (23) My thoughts jump from topic to topic & 24.7 & 13.2 & 40.0 & 8.791 & 1 & .003 \\
\hline (24) I do things more quickly and/or more easily & 57.0 & 35.8 & 85.0 & 22.467 & 1 & $<.001$ \\
\hline (25) I am more impatient and/or get irritable more easily & 30.1 & 18.9 & 45.0 & 7.398 & 1 & .007 \\
\hline (26) I can be exhausting or irritating for others & 20.4 & 13.2 & 30.0 & 3.954 & 1 & .047 \\
\hline (27) I get into more quarrels & 19.4 & 15.1 & 25.0 & 1.433 & 1 & ns \\
\hline (28) My mood is higher, more optimistic & 68.8 & 52.8 & 90.0 & 14.676 & 1 & $<.001$ \\
\hline (29) I drink more coffee & 19.4 & 13.2 & 27.5 & 2.983 & 1 & ns \\
\hline (30) I smoke more cigarettes & 15.1 & 5.7 & 27.5 & 8.503 & 1 & .004 \\
\hline (31) I drink more alcohol & 7.5 & 1.9 & 15.0 & 5.632 & 1 & .018 \\
\hline (32) I take more drugs (sedatives, anxiolytics, stimulants) & 7.5 & 5.7 & 10.0 & 0.617 & 1 & ns \\
\hline
\end{tabular}

*Standard cut-off: endorsement of at least 14 or more items.

List of abbreviations: HCL-32: hypomania checklist; Chi-sq: chi-square test; df: degrees of freedom; ns: nonsignificant.

on the prevalence of $\mathrm{BD}$ in depressed patients of primary care [11]. On the contrary, HCL-32 appears to be more sensitive in detecting subthreshold hypomanic conditions, identifying more than one-third of patients as cases, but this characteristic might not be compatible with the purpose in primary care of a close examination of all patients that screen positive. The population identified by MDQ and psychometric proprieties of the scale suggests higher accuracy of the instrument compared to HCL-32. Besides, MDQ shows a good feasibility: it is shorter compared to HCL-32 and a lower cut-off simplifies the scoring even further. These proprieties make MDQ accessible and usable in a primary care service and might aid PCP in order to better recognize $\mathrm{BD}$ in patients presenting depressive symptoms.

An interesting finding emerged from the present study in a subsample. Patients who reported more hypomanic symptoms with a higher total score of the screening instruments correlated with higher total score for current depressive symptoms evaluated with the PHQ-9. We hypothesized that this result, reported also by Smith et al. [24], might be due to a subjective overestimation of previous well-being compared to the current depressive state, but it could also reflect the more severe condition of bipolar than unipolar depression [41]. As PHQ-9 is widespread in primary care, we could hypothesize 
TABLE 5: Demographic and clinical characteristics according to threshold score on the MDQ.

\begin{tabular}{|c|c|c|c|c|c|c|c|c|}
\hline & \multicolumn{3}{|c|}{ MDQ: standard cut-off* } & \multicolumn{5}{|c|}{ MDQ: cut-off $6^{* *}$} \\
\hline & Positive & Negative & $\begin{array}{c}F / \text { chi-square } \\
\text { df }\end{array}$ & $P$ & Positive & Negative & $\begin{array}{c}F / \text { chi-square } \\
\text { df }\end{array}$ & $P$ \\
\hline Gender, women, \% & 62.5 & 72.9 & $.402,1$ & ns & 63.6 & 74.6 & $1.022,1$ & ns \\
\hline Age, years: mean \pm sd & $48.4 \pm 11.8$ & $49.1 \pm 15.4$ & $.018,1$ & ns & $45.5 \pm 13.9$ & $50.2 \pm 15.3$ & $1.608,1$ & ns \\
\hline Education level, high, \% & 62.5 & 65.1 & $.833,1$ & ns & 77.3 & 60.9 & $2.019,1$ & ns \\
\hline Civil status, separated/divorced, $\%$ & 25.0 & 20.0 & $.461,2$ & ns & 31.8 & 16.9 & $2.326,2$ & ns \\
\hline Occupation, nonprofessional condition, $\%$ & 37.5 & 46.4 & $.247,1$ & ns & 40.9 & 47.1 & $.276,1$ & ns \\
\hline Current therapy, $\mathrm{AD}, \%$ & 62.5 & 67.1 & $.074,1$ & ns & 45.5 & 73.2 & $5.87,2$ & .015 \\
\hline Panic attack disorder, \% & 37.5 & 10.6 & $4.757,1$ & .029 & 18.2 & 11.3 & $.723,1$ & ns \\
\hline Smoking habit, yes, $\%$ & 62.5 & 25.9 & $4.859,1$ & .028 & 42.9 & 25 & $2.459,1$ & ns \\
\hline Cardiovascular risk factors, 2 or more, $\%$ & 37.5 & 10.6 & $4.757,1$ & .029 & 22.7 & 9.9 & $2.499,1$ & ns \\
\hline
\end{tabular}

*Standard cut-off: endorsement of at least 7 or more symptoms items, cooccurrence of two or more symptoms, and moderate or severe impairment.

${ }^{* *}$ Cut-off 6: endorsement of at least 6 or more symptoms items, with no adjunctive criteria.

List of abbreviations: MDQ: mood disorder questionnaire; Chi-sq: chi-square test; df: degrees of freedom; sd: standard deviation; ns: nonsignificant; AD: antidepressant.

TABLE 6: Demographic and clinical characteristics according to threshold score on the HCL-32.

\begin{tabular}{|c|c|c|c|c|c|c|c|c|}
\hline & \multicolumn{3}{|c|}{ HCL-32: Standard cut-off* } & \multicolumn{5}{|c|}{ HCL-32: cut-off $12^{* *}$} \\
\hline & Positive & Negative & $\begin{array}{c}F / \text { chi-Square } \\
\text { df }\end{array}$ & $P$ & Positive & Negative & $\begin{array}{c}\text { F/chi-Square, } \\
\text { df }\end{array}$ & $P$ \\
\hline Gender, women \% & 72.5 & 71.7 & $.011,1$ & ns & 71.2 & 73.2 & $.051,1$ & ns \\
\hline Age, years: mean \pm sd & $45.7 \pm 13.6$ & $51.6 \pm 15.6$ & $3.457,1$ & ns & $46.0 \pm 14.2$ & $52.9 \pm 15.3$ & $4.92,1$ & .029 \\
\hline Education level, high \% & 76.9 & 55.8 & $5.273,1$ & .037 & 78.4 & 47.5 & $9.34,1$ & .002 \\
\hline Civil Status, separated/divorced \% & 30.0 & 13.2 & $5.638,2$ & ns & 25.0 & 14.6 & $2.124,2$ & $\mathrm{~ns}$ \\
\hline Occupation, non professional condition $\%$ & 42.5 & 48.1 & $.301,1$ & ns & 42.3 & 50.0 & $.560,1$ & ns \\
\hline Current therapy, $\mathrm{AD} \%$ & 57.5 & 73.6 & $2.674,1$ & ns & 44.2 & 19.5 & $6.343,1$ & .012 \\
\hline Panic attack disorder \% & 12.5 & 13.2 & $.012,1$ & ns & 13.5 & 12.2 & $.035,1$ & ns \\
\hline Smoking habit, yes \% & 42.1 & 19.6 & $5.273,1$ & .022 & 38.8 & 17.5 & $4.83,1$ & .028 \\
\hline Cardiovascular risk factors, 2 or more $\%$ & 20.0 & 7.5 & $3.176,1$ & ns & 15.4 & 9.8 & $.654,1$ & ns \\
\hline
\end{tabular}

*Standard cut-off: endorsement of at least 14 or more items.

${ }^{* *}$ Cut-off 12: endorsement of at least 12 or more items.

List of abbreviations: HCL-32: hypomania checklist; Chi-sq: chi-square test; df: degrees of freedom; sd: standard deviation; ns: non significant; AD: antidepressant.

a two-stage strategy to refine the mood disorders assessment. In particular, PCPs should also administer MDQ to patients reporting high PHQ-9 score.

4.1. Limitations. The present study has several limitations. The initial assessment of mood disorder was done by PCPs, and there is the possibility of a misdiagnosis of depression. Subsequently the diagnosis of mood disorder was performed by a consultant psychiatrist following DSM-IV criteria, but without a structured diagnostic interview like the Structured Clinical Interview for DSM-IV Axis I Disorders (SCID-I) [42]. As a consequence, we admit a risk of selection of a highly heterogeneous sample and a possible under or overestimate of the diagnosis of $\mathrm{BD}$; however, our symptoms prevalence and our values of sensitivity and specificity seem in line with previous literature on this topic $[18,19]$.

Furthermore, the sample was relatively small, and we gathered data to perform ROC analysis only for 67 patients; therefore, our results need to be interpreted with caution and further researches including studies with higher sample sizes are needed.

Finally, we did not collect data about excluded patients and those that refused participation in the study or psychiatric consultation; however, the recruitment was performed in the busy setting of the primary care, and we chose to adopt a simple and easy procedure to avoid a supplementary work for PCPs.

\section{Conclusion}

PCP is often the first health contact for assessment and treatment of patients with depressive symptoms. Despite programs of training and collaboration aimed to increase the PCP's ability to detect depressive disorders, differential diagnosis of a bipolar depression remains still a difficult and 


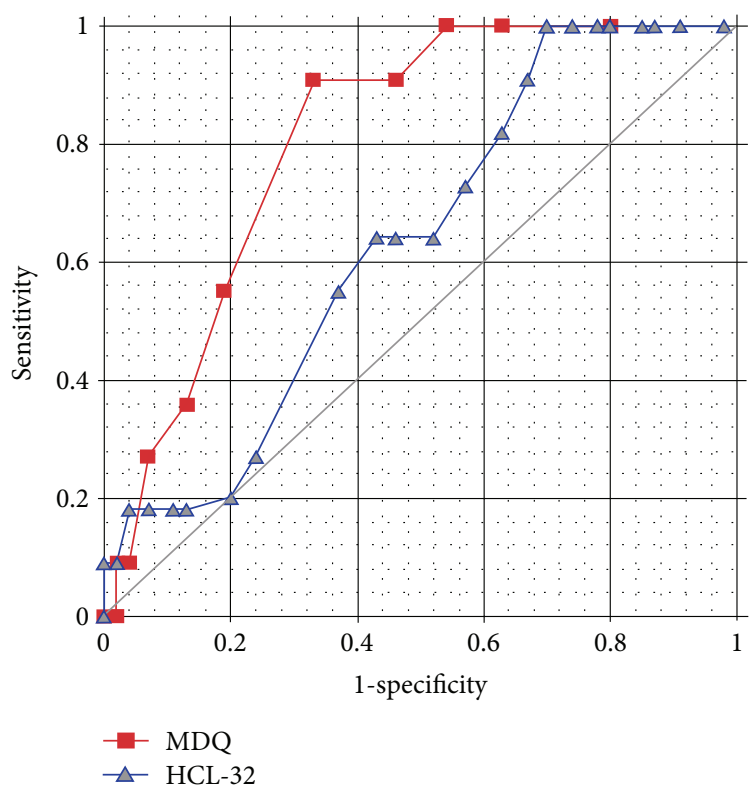

\begin{tabular}{llllllllllllll}
\hline $\begin{array}{l}\text { MDQ } \\
\text { Cut-off }\end{array}$ & 1 & 2 & 3 & 4 & 5 & 6 & 7 & 8 & 9 & 10 & 11 & 12 & 13 \\
\hline Sensitivity & 1.00 & 1.00 & 1.00 & .91 & .91 & .55 & .36 & .27 & .09 & .09 & .00 & .00 & .00 \\
Specificity & .20 & .37 & .46 & .54 & .67 & .81 & .87 & .93 & .96 & .98 & .98 & 1.00 & 1.00 \\
1-specificity & .80 & .63 & .54 & .46 & .33 & .19 & .13 & .07 & .04 & .02 & .02 & .00 & .00 \\
\hline
\end{tabular}

\begin{tabular}{|c|c|c|c|c|c|c|c|c|c|c|c|c|c|c|c|c|}
\hline $\begin{array}{l}\text { HCL-32 } \\
\text { Cut-off }\end{array}$ & 1 & 2 & 3 & 4 & 5 & 6 & 7 & 8 & 9 & 10 & 11 & 12 & 13 & 14 & 15 & 16 \\
\hline Sensitivity & 1.00 & 1.00 & 1.00 & 1.00 & 1.00 & 1.00 & 1.00 & 1.00 & 1.00 & .91 & .82 & .73 & .64 & .64 & .64 & .55 \\
\hline Specificity & .02 & .09 & .13 & .15 & .20 & .20 & .22 & .26 & .30 & .33 & .37 & .43 & .48 & .54 & .57 & .63 \\
\hline 1-specificity & .98 & .91 & .87 & .85 & .80 & .80 & .78 & .74 & .70 & .67 & .63 & .57 & .52 & .46 & .43 & .37 \\
\hline
\end{tabular}

\begin{tabular}{|c|c|c|c|c|c|c|c|c|c|c|c|c|c|c|c|c|}
\hline $\begin{array}{l}\text { HCL-32 } \\
\text { Cut-off }\end{array}$ & 17 & 18 & 19 & 20 & 21 & 22 & 23 & 24 & 25 & 26 & 27 & 28 & 29 & 30 & 31 & 32 \\
\hline Sensitivity & .27 & .18 & .18 & .18 & .18 & .18 & .09 & .09 & .09 & .00 & .00 & .00 & .00 & .00 & .00 & .00 \\
\hline Specificity & .76 & .80 & .87 & .89 & .93 & .96 & .98 & 1.00 & 1.00 & 1.00 & 1.00 & 1.00 & 1.00 & 1.00 & 1.00 & 1.00 \\
\hline 1-specificity & .24 & .20 & .13 & .11 & .07 & .04 & .02 & .00 & .00 & .00 & .00 & .00 & .00 & .00 & .00 & .00 \\
\hline
\end{tabular}

FIGURE 1: ROC Analysis of the performance of MDQ and HCL-32 in the sample.

complex task. This background supports the use of specific instruments that can raise diagnostic accuracy of PCPs.

In the present study, MDQ showed acceptable proprieties as screening instrument, with better psychometric characteristics using 5 as cut-off. MDQ appears to be more specific, easier and shorter than HCL-32, and it also takes little time to score. These features fit well in the busy setting of primary care, where PCPs have little time to dedicate to assessment. It cannot solve the diagnostic doubt between unipolar and bipolar depression, but it is the chance of having more patient's information during the visit to make correct therapeutic choices or to proceed in further investigations or refer to mental health services.

\section{Acknowledgment}

We thank all primary care physicians referring patients for this study. 


\section{References}

[1] S. Pini, V. De Queiroz, D. Pagnin et al., "Prevalence and burden of bipolar disorders in European countries," European Neuropsychopharmacology, vol. 15, no. 4, pp. 425-434, 2005.

[2] K. R. Merikangas, M. Ames, L. Cui et al., "The impact of comorbidity of mental and physical conditions on role disability in the US adult household population," Archives of General Psychiatry, vol. 64, no. 10, pp. 1180-1188, 2007.

[3] M. Raja and A. Azzoni, "Suicide attempts: differences between unipolar and bipolar patients and among groups with different lethality risk," Journal of Affective Disorders, vol. 82, no. 3, pp. 437-442, 2004.

[4] P. B. Mitchell and G. S. Malhi, "Bipolar depression: phenomenological overview and clinical characteristics," Bipolar Disorders, vol. 6, no. 6, pp. 530-539, 2004.

[5] F. Benazzi, "Bipolar disorder-focus on bipolar II disorder and mixed depression," The Lancet, vol. 369, no. 9565, pp. 935-945, 2007.

[6] B. M. Müller-Oerlinghausen, A. Berghöfer, and M. Bauer, "Bipolar disorder," The Lancet, vol. 359, no. 9302, pp. 241-247, 2002.

[7] L. L. Judd, H. S. Akiskal, P. J. Schettler et al., "A prospective investigation of the natural history of the long-term weekly symptomatic status of bipolar II disorder," Archives of General Psychiatry, vol. 60, no. 3, pp. 261-269, 2003.

[8] R. W. Kupka, L. L. Altshuler, W. A. Nolen et al., "Three times more days depressed than manic or hypomanic in both bipolar I and bipolar II disorder," Bipolar Disorders, vol. 9, no. 5, pp. 531535, 2007.

[9] R. M. A. Hirschfeld, L. Lewis, and L. A. Vornik, "Perceptions and impact of bipolar disorder: how far have we really come? Results of the national depressive and manic-depressive association 2000 survey of individuals with bipolar disorder," Journal of Clinical Psychiatry, vol. 64, no. 2, pp. 161-174, 2003.

[10] J. Angst, "Do many patients with depression suffer from bipolar disorder?” Canadian Journal of Psychiatry, vol. 51, no. 1, pp. 3-5, 2006.

[11] S. L. Dubovsky, K. Leonard, K. Griswold et al., "Bipolar disorder is common in depressed primary care patients," Postgraduate Medicine, vol. 123, no. 5, pp. 129-133, 2011.

[12] M. A. Frye, J. R. Calabrese, M. L. Reed et al., "Use of health care services among persons who screen positive for bipolar disorder," Psychiatric Services, vol. 56, no. 12, pp. 1529-1533, 2005.

[13] R. M. A. Hirschfeld, J. B. W. Williams, R. L. Spitzer et al., "Development and validation of a screening instrument for bipolar spectrum disorder: the mood disorder questionnaire," The American Journal of Psychiatry, vol. 157, no. 11, pp. 18731875, 2000.

[14] J. Angst, R. Adolfsson, F. Benazzi et al., "The HCL-32: Towards a self-assessment tool for hypomanic symptoms in outpatients," Journal of Affective Disorders, vol. 88, no. 2, pp. 217-233, 2005.

[15] National Collaborating Centre for Mental Health (UK), Bipolar Disorder: The Management of Bipolar Disorder in Adults, Children and Adolescents, in Primary and Secondary Care, British Psychological Society, Leicester, UK, 2006.

[16] E. Gorski and K. C. Willis, "A pilot study examining the relationship between patients' complaints and scores on the hirschfeld MDQ," Primary Care Companion To the Journal of Clinical Psychiatry, vol. 5, no. 5, pp. 201-204, 2003.
[17] E. Gorski, D. Ghezzi, and K. C. Willis, "Factors associated with positive screens on the mood disorder questionnaire in primary care," Primary Care Companion to the Journal of Clinical Psychiatry, vol. 8, no. 5, pp. 264-268, 2006.

[18] A. K. Das, M. Olfson, M. J. Gameroff et al., "Screening for bipolar disorder in a primary care practice," The Journal of the American Medical Association, vol. 293, no. 8, pp. 956-963, 2005.

[19] F. Rouillon, I. Gasquet, R. P. Garay, and S. Lancrenon, "Screening for bipolar disorder in patients consulting general practitioners in France," Journal of Affective Disorders, vol. 130, no. 3, pp. 492-495, 2011.

[20] R. M. A. Hirschfeld, A. R. Cass, D. C. L. Holt, and C. A. Carlson, "Screening for bipolar disorder in patients treated for depression in a family medicine clinic," The Journal of the American Board of Family Practice, vol. 18, no. 4, pp. 233-239, 2005.

[21] M. Olfson, A. K. Das, M. J. Gameroff et al., "Bipolar depression in a low-income primary care clinic," American Journal of Psychiatry, vol. 162, no. 11, pp. 2146-2151, 2005.

[22] J. F. Chiu and P. R. Chokka, "Prevalence of bipolar disorder symptoms in primary care (ProBiD-PC): a Canadian study," Canadian Family Physician, vol. 57, no. 2, pp. e58-e67, 2011.

[23] S. N. Ghaemi, C. J. Miller, D. A. Berv, J. Klugman, K. J. Rosenquist, and R. W. Pies, "Sensitivity and specificity of a new bipolar spectrum diagnostic scale," Journal of Affective Disorders, vol. 84, no. 2-3, pp. 273-277, 2005.

[24] D. J. Smith, E. Griffiths, M. Kelly, K. Hood, N. Craddock, and S. A. Simpson, "Unrecognised bipolar disorder in primary care patients with depression," British Journal of Psychiatry, vol. 199, no. 1, pp. 49-56, 2011.

[25] M. G. Carta, M. C. Hardoy, M. Cadeddu et al., "The accuracy of the Italian version of the hypomania checklist (HCL-32) for the screening of bipolar disorders and comparison with the mood disorder questionnaire (MDQ) in a clinical sample," Clinical Practice and Epidemiology in Mental Health, vol. 2, article 2, 2006.

[26] E. Vieta, J. Sánchez-Moreno, A. Bulbena et al., "Cross validation with the mood disorder questionnaire (MDQ) of an instrument for the detection of hypomania in Spanish: the 32 item hypomania symptom check list (HCL-32)," Journal of Affective Disorders, vol. 101, no. 1-3, pp. 43-55, 2007.

[27] J. K. Rybakowski, J. Angst, D. Dudek et al., "Polish version of the hypomania checklist (HCL-32) scale: the results in treatment-resistant depression," European Archives of Psychiatry and Clinical Neuroscience, vol. 260, no. 2, pp. 139-144, 2010.

[28] T. D. Meyer, B. Bernhard, C. Born et al., "The Hypomania Checklist-32 and the MDQ as screening tools-going beyond samples of purely mood-disordered patients," Journal of Affective Disorders, vol. 128, no. 3, pp. 291-298, 2011.

[29] Y. Poon, K. F. Chung, K. C. Tso, C. L. Chang, and D. Tang, “The use of mood disorder questionnaire, hypomania checklist-32 and clinical predictors for screening previously unrecognised bipolar disorder in a general psychiatric setting," Psychiatry Research, vol. 195, no. 3, pp. 111-117, 2011.

[30] C. C. Chou, I. H. Lee, T. L. Yeh et al., "Comparison of the validity of the chinese versions of the hypomania symptom checklist32 (HCL-32) and mood disorder questionnaire (MDQ) for the detection of bipolar disorder in medicated patients with major depressive disorder," International Journal of Psychiatry in Clinical Practice, vol. 16, no. 2, pp. 132-137, 2012. 
[31] R. L. Spitzer, K. Kroenke, and J. B. W. Williams, "Validation and utility of a self-report version of PRIME-MD: the PHQ primary care study," The Journal of the American Medical Association, vol. 282, no. 18, pp. 1737-1744, 1999.

[32] M. C. Hardoy, M. Cadeddu, A. Murru et al., "Validation of the Italian version of the "Mood Disorder Questionnaire" for the screening of bipolar disorders," Clinical Practice and Epidemiology in Mental Health, vol. 1, article 8, 2005.

[33] J. A. Sweet, "Operating characteristic analysis," Psychology Science, vol. 5, pp. 990-1000, 1975.

[34] C. de Dios, E. Ezquiaga, A. García, J. M. Montes, C. Avedillo, and B. Soler, "Usefulness of the Spanish version of the mood disorder questionnaire for screening bipolar disorder in routine clinical practice in outpatients with major depression," Clinical Practice and Epidemiology in Mental Health, vol. 4, article 14, 2008.

[35] B. Kim, H. R. Wang, J. I. Son, C. Y. Kim, and Y. H. Joo, "Bipolarity in depressive patients without histories of diagnosis of bipolar disorder and the use of the mood disorder questionnaire for detecting bipolarity," Comprehensive Psychiatry, vol. 49, no. 5, pp. 469-475, 2008.

[36] P. Bech, E. M. Christensen, M. Vinberg, G. Bech-Andersen, and L. V. Kessing, "From items to syndromes in the hypomania checklist (HCL-32): psychometric validation and clinical validity analysis," Journal of Affective Disorders, vol. 132, no. 1-2, pp. 48-54, 2011.

[37] L. Forty, D. Smith, L. Jones et al., "Identifying hypomanic features in major depressive disorder using the hypomania checklist (HCL-32)," Journal of Affective Disorders, vol. 114, no. 1-3, pp. 68-73, 2009.

[38] J. R. Calabrese, D. J. Muzina, D. E. Kemp et al., "Predictors of bipolar disorder risk among patients currently treated for major depression," MedGenMed Medscape General Medicine, vol. 8, no. 3, p. 38, 2006.

[39] K. J. Tsuchiya, M. Byrne, and P. B. Mortensen, "Risk factors in relation to an emergence of bipolar disorder: a systematic review," Bipolar Disorders, vol. 5, no. 4, pp. 231-242, 2003.

[40] A. Kreinin, D. Novitski, D. Rabinowitz, A. Weizman, and A. Grinshpoon, "Association between tobacco smoking and bipolar affective disorder: clinical, epidemiological, cross-sectional, retrospective study in outpatients," Comprehensive Psychiatry, vol. 53, no. 3, pp. 269-274, 2011.

[41] G. M. Goodwin, I. Anderson, C. Arango et al., "ECNP consensus meeting. Bipolar depression. Nice, March 2007," European Neuropsychopharmacology, vol. 18, no. 7, pp. 535-549, 2008.

[42] M. B. First, R. L. Spitzer, M. Gibbon, and J. B. W. Williams, Structured Clinical Interview For DSM-IV Axis I Disorders, Clinician Version (SCID-CV), American Psychiatric Press, Washington, DC, USA, 1996. 


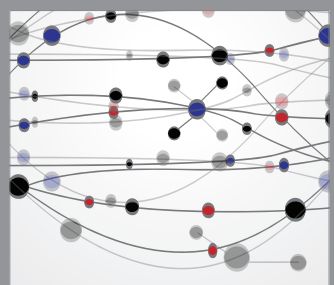

The Scientific World Journal
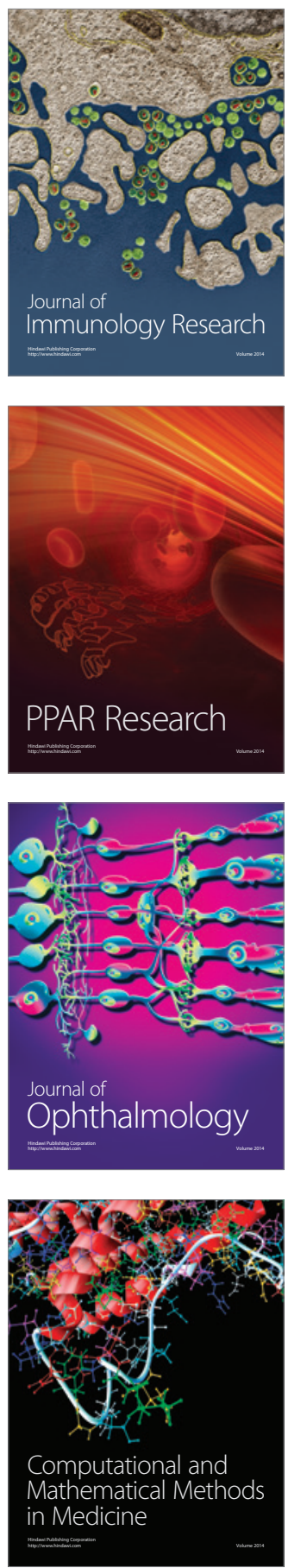

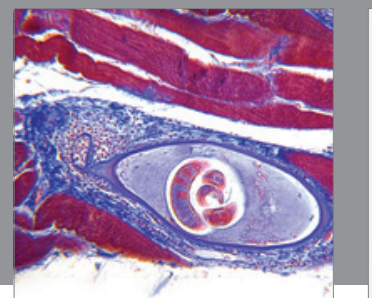

Gastroenterology

Research and Practice
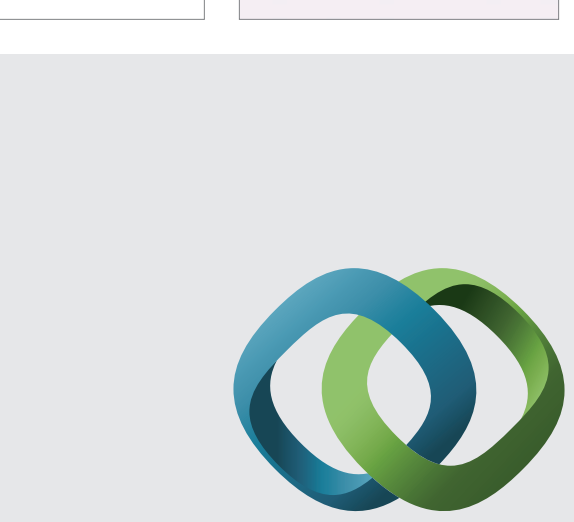

\section{Hindawi}

Submit your manuscripts at

http://www.hindawi.com
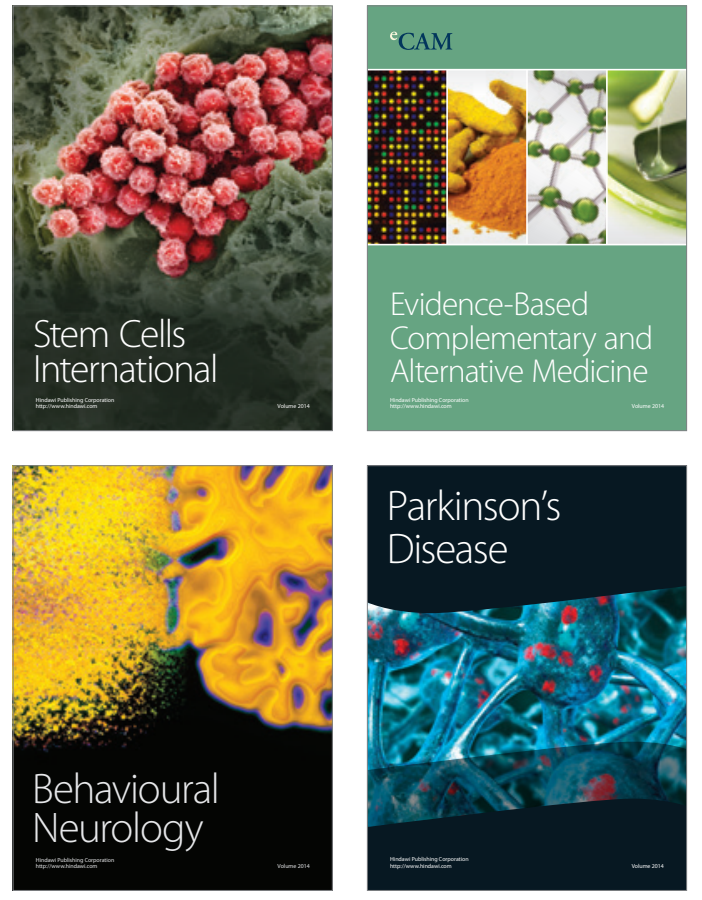
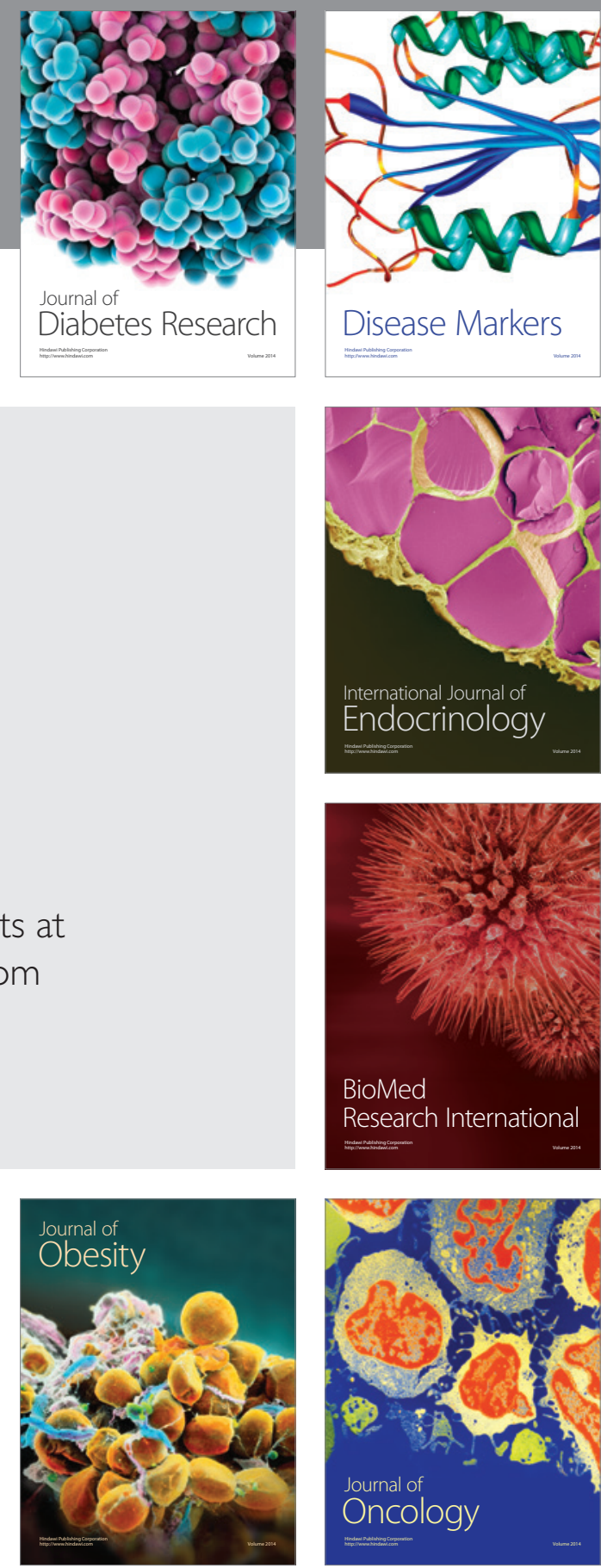

Disease Markers
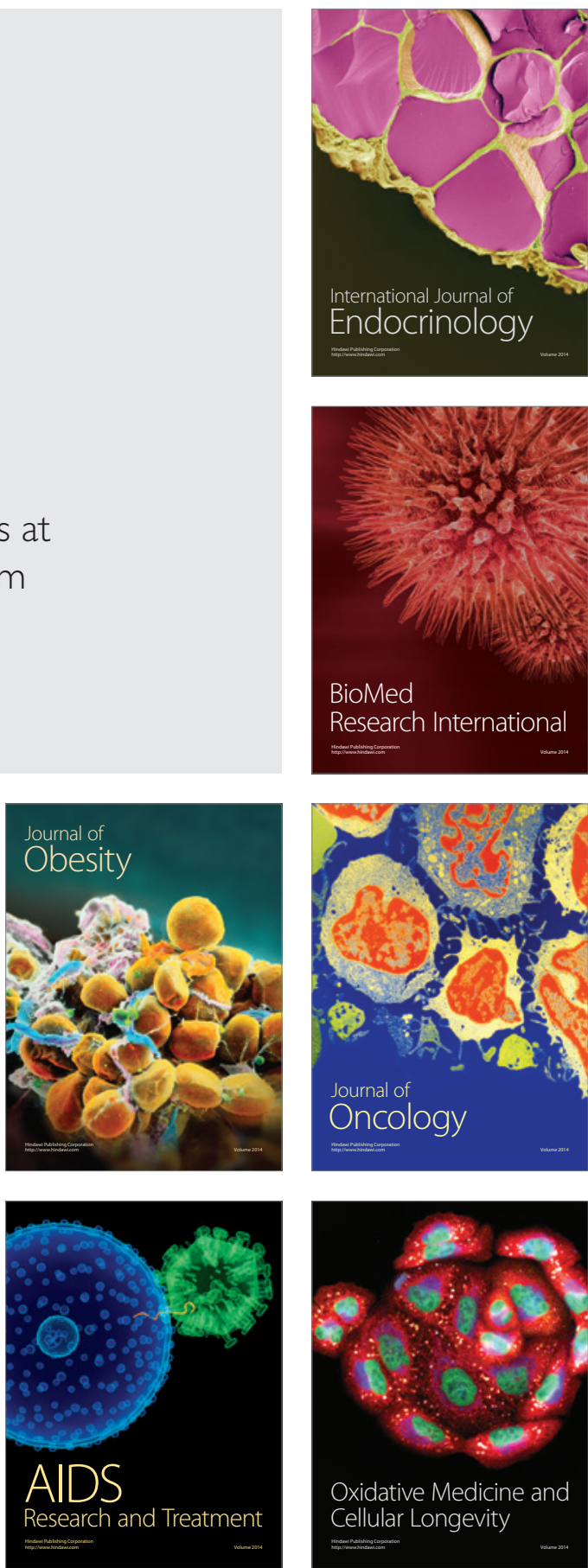